\title{
Une brèche dans les masques de théâtre : apparitions des visages et des corps âgés dans les créations de la Societas Raffaello Sanzio
}

Tearing Theatre Masks: Apparitions of Old Faces and Bodies in Societas Raffaello Sanzio's Works

\section{Erica Magris}

\section{OpenEdition Journals}

Édition électronique

URL : https://journals.openedition.org/recherchestravaux/740

DOI : 10.4000/recherchestravaux.740

ISSN : 1969-6434

\section{Éditeur}

UGA Éditions/Université Grenoble Alpes

\section{Édition imprimée}

Date de publication : 28 mai 2015

Pagination : 93-104

ISBN : 978-2-84310-298-1

ISSN : 0151-1874

\section{Référence électronique}

Erica Magris, « Une brèche dans les masques de théâtre : apparitions des visages et des corps âgés dans les créations de la Societas Raffaello Sanzio », Recherches \& Travaux [En ligne], 86 | 2015, mis en ligne le 28 novembre 2016, consulté le 29 octobre 2021. URL : http://journals.openedition.org/ recherchestravaux/740; DOI : https://doi.org/10.4000/recherchestravaux.740 
Erica MAGRIS

Université Paris 8

EA «Scènes du monde, création, savoirs critiques»

\section{Une brèche dans les masques de théâtre : apparitions des visages et des corps âgés dans les créations de la Socìetas Raffaello Sanzio}

Sur le vase de Pronomos, l'un des témoignages figuratifs les plus importants du théâtre grec, le monde des dieux et le monde des hommes, représentés de part et d'autre des anses, sont unis par le geste d'offrande d'un Satyre, qui établit le lien entre le rituel et la représentation : d'un côté, le dynamisme des danses effrénées des Bacchantes et des Satyres autour de Dionysos et Ariane, élancés à l'avant; de l'autre l'immobilité des deux mêmes personnages, assis sur une banquette en attendant le début du spectacle, préparé par de nombreux choreutes, quatre acteurs, un poète, des musiciens ${ }^{\mathrm{I}}$. Le théâtre se définit donc comme le lieu du regard, où le travail artistique collectif reproduit la métamorphose engendrée par l'ivresse divine. Pourtant l'homme, même s'il se rapproche du dieu, ne peut que représenter cette transformation, par l'outil fondamental du masque, que les choreutes et les acteurs tiennent dans les mains. Ces masques portent les marques d'une différence substantielle entre le monde divin et le monde humain : ceux des choreutes, représentant le cortège de Satyres de Dionysos avec leurs attributs animaux, sont tous pareils et montrent des visages barbus; ceux des acteurs, au contraire, sont individualisés et indiquent des personnages différents, caractérisés par leur sexe et surtout par leur âge : la reine, le jeune héros, le tyran d'âge mûr et le vieillard, avec sa barbe blanche et ses cheveux longs. Même s'il se veut d'origine divine et

I. Pour l'interprétation de vase, nous faisons référence à F. Mastropasqua, «Il cratere di Pronomos", dans Metamorfosi del teatro, Naples, Edizioni Scientifiche Italiane, 1998, p. 39-56. 
mystérique, capable de mettre en œuvre une altérité spatio-temporelle fondée sur la transformation des participants à l'action scénique, le théâtre demeure ancré dans la condition humaine, dont il révèle le caractère transitoire et fragile lié au flux du temps qu'il ne peut pas arrêter.

L'interrogation du tragique et de l'origine rituelle du théâtre, ainsi que la mise en place de tensions oxymoriques dans les relations entre matérialité et image, entre réel et construction d'un univers autre, onirique et révélateur, sont au cour de la poétique scénique de la Sociètas Raffaello Sanzio et de Romeo Castellucci. À partir de La Discesa di Inanna, spectacle réalisé en 1989, inspiré du mythe sumérien qui narre la descente de la déesse de la fertilité dans l'au-delà, ces éléments de recherche influencent le choix des acteurs et la présence d'âges différents sur scène. Comme le raconte le critique Oliviero Ponte Di Pino, les alors jeunes fondateurs de la compagnie - Chiara et Paolo Guidi, Claudia et Romeo Castellucci - sont accompagnés à cette occasion par des figures de l'altérité : Giunta Biserna, mère des Castellucci, «actrice "naturelle" et présence dissonante», deux petites filles «identiques et jumelles, qui, par leur incohérente ambiguïté, témoigneront des horreurs sans noms du royaume des ombres $»^{2}$, et des animaux - des brebis, des chèvres et une famille de babouins. Depuis, des présences liminaires situées aux points extrêmes de la vie - les vieillards, les enfants, les malades - ou étrangères à l'homme - animaux, automates habitent régulièrement les créations de la Raffaello Sanzio, et, comme dans La Discesa di Inanna, évoquent sur scène le non-vivant, le non-humain, mais aussi le trop-humain.

À la différence du vase de Pronomos, ces figures ne surgissent pas de la métamorphose engendrée par le masque, mais sont des présences vraies, authentiques, brutes : non seulement des visages, mais des corps, exhibés avec leurs blessures et leurs faiblesses. Ces corps, comme l'explique Bénédicte Boisson à propos de Giulio Cesare ${ }^{3}$, réduits à leur pure existence, sont insérés dans un dispositif signifiant flottant producteur d'images, une «matrice» qui leur donne une dimension esthétique et symbolique. Ce sont des acteurs figurants, qui revêtent une fonction ostentatoire et supportent le scandale de se montrer sur scène. Dans un texte de 1994, intitulé «Acteur, ton nom n’est pas exact ${ }^{4}$,, Romeo Castellucci écrit que

2. O. Ponte Di Pino, Il teatro di Romeo Castellucci e della Societas Raffaello Sanzio, e-book publié par Doppiozero et Ateatro, 2013, p. 39.

3. B. Boisson, «Le théâtre ou l'exhibition du monstre. La mise en scène des corps stigmatisés dans Giulio Cesare de Romeo Castellucci ", dans L'Annuaire théâtral : revue québécoise d'études théâtrales, ${ }^{\circ} 37,2005$, p. 183-196.

4. Récemment R. Castellucci a créé des performances qui portent ce même titre. 
Quand on monte sur scène, on est, d'emblée, coupable. Être sur une scène signifie recevoir, de façon masochiste, une punition; être sur une scène est déjà un état de merveille et de honte : la honte d'être pris là sur le fait. [...] En effet, c'est la honte, le nœud essentiel de l'exposition. L'ostension est déjà lapidation de regards. La scène devient alors un lieu de précipitation et de seuil; un lieu où la souffrance ostensoir coïncide avec le regard. La scène restitue la limite du corps et le renvoie à sa propre limite - à découvert - qui est souffrances.

Les corps vieux ou vieillissants répondent à une telle conception de l'acteur et de son rôle scénique. Le sens ultime de leur présence demeure constant de Inanna à Il senso del volto di Cristo : en exhibant les marques du temps et le poids des expériences de la vie, insérés dans un réseau d'associations et d'oppositions avec les autres «présences scandaleuses» de la scène, ils transgressent les tabous de la société occidentale contemporaine et mettent les spectateurs face à l'inéluctabilité du déclin physique, la perte de l'intégrité et l'anticipation de la mort qui nous attend tous ${ }^{6}$. Néanmoins, des articulations de sens plus précises, des constantes et des variations émergent de chaque création, ainsi que des renvois intertextuels d'un spectacle à l'autre. Deux lignées en particulier se dessinent des années I990 à aujourd'hui, liées aux archétypes maternel et paternel : d'une part, la vieillesse féminine, de l'autre, la vieillesse masculine dans le cadre de la relation père-fils.

\section{La vieillesse féminine : l'union de génération et mort}

Les premiers parents de l'humanité font leur apparition dans le «musée du sommeil7 » déployé dans Genesi (I999) : d'abord, Adam, incarné par un contorsionniste, dont le corps semble un enchevêtrement difforme de chair et de membres; ensuite Ève, condamnée par Dieu à enfanter dans la douleur. Enfermée derrière une vitrine, entourée par des animaux empaillés et des organes pendus aux cintres, elle soupire et se plaint ${ }^{8}$. Après quelques minutes, elle sort et, à petits pas incertains, s'avance sur scène, éclairée par une lumière

5. R. Castellucci, «Acteur, ton nom n'est pas exact», dans C. et R. Castellucci, Les Pèlerins de la matière. Théorie est praxis du théâtre, trad. K. Espinosa, Besançon, Les Solitaires Intempestifs, 200I, p. 32-33.

6. La dimension centrale du thème du vieillissement et de la mort dans la poétique de la Raffaello Sanzio se manifeste aussi dans le choix de la couverture du livre Epitaph : une image en noir et blanc du masque funèbre de l'artiste allemand Christian Rauch, les yeux fermés et noircis, les joues creuses et la bouche édentée. Voir Socìetas Raffaello Sanzio et R. Castellucci, Epitaph, Milan-Besançon, Ubulibri - Les Solitaires Intempestifs, 2003.

7. Genesi. From the museum of sleep est le titre complet du spectacle.

8. Eve est interprétée par M. L. Cantarella. 
froide dans la semi-obscurité environnante. Toute nue, elle se place face au public, debout, en essayant de cacher tant bien que mal sa poitrine et ses parties intimes avec ses bras et ses mains. Sa figure et sa posture rappellent la fresque Adam et Ėve chassés du paradis peinte par Masaccio dans la chapelle Brancacci de l'église Santa Maria del Carmine à Florence (I426-I427), une source d'inspiration fondamentale pour Romeo Castellucci, mentionnée dans le programme'. Mais, si dans la fresque le désespoir d'Ève s'exprime surtout par son visage, sa bouche ouverte et ses yeux fermés et noircis, dans le spectacle, ce désespoir se manifeste dans la totalité de son corps vieillissant et mutilé horriblement par une mastectomie, dans le ventre gras et flasque, dans ses longs cheveux - peut-être blonds -, qui apparaissent blancs sous le faisceau du projecteur. Son visage, comme le dit Katia Arfara, "pétrifié avant le cri, [...] est une "force vide", un "champ de mort" qui n'a pas encore trouvé ses propres traits (Artaud) ${ }^{\mathrm{I}}{ }^{\prime}$. Après s'être exposée et débattue dans la honte et la détresse, Ève enlève sa perruque, découvrant sa tête chauve, et commence des opérations pour se lier aux organes accrochés, à l'issue desquelles elle se trouve allongée, immobile et ligotée par les câbles, tandis que le plateau se soulève dans une sorte de respiration. La première mère de l'humanité se transforme ainsi en malade au corps souffrant, emprisonnée dans une vie artificielle et médicalisée.

La même association entre femme, vieillesse et univers médical se retrouve dans une séquence de l'épisode \#os Bergen de la Tragedia Endogonidia, interprétée par la même actrice. Au début du spectacle, se profile dans l'obscurité enveloppant la scène, une figure de mère vieillie ${ }^{\text {II }}$, assise de dos sur une table, face à un rideau en plastique transparent. Elle ne porte qu'une culotte blanche, et elle a les cheveux assez longs, gris : seuls son dos et ses pieds sont visibles, tandis que son visage reste inconnu et mystérieux. La médecine est évoquée par un appareil aux tuyaux transparents posé à sa droite et par un

9. R. Castellucci, «Genesi. From the Museum of Sleep. Le programme», dans C. et R. Castellucci, Les Pèlerins de la matière, ouvr. cité, p. I26.

IO. K. Arfara, Théâtralités contemporaines : entre les arts plastiques et les arts de la scène, Berne, Peter Lang, 20II, p. 298.

II. Comme le soulignent E. Pitozzi et A Sacchi, la figure de la Mère est récurrente dans le cycle: «La Mère semble être la figure dans laquelle le monde de la scène se reconnaît comme créature, et créature qui conçoit, qui est origine de la vie et donc de la matière pour la Tragedia Endogonidia. [...] La Mère se rend disponible à de multiples formes; elle s'ouvre, littéralement, au devenir : de femme enceinte elle régresse à l'état de foetus, renait sous la forme d'une enfant, devient homme, selon un principe de variations continuelles qui redouble le principe de fuite, propre à la Tragedia Endogonidia" (dans E. Pitozzi et A. Sacchi, Itinera : trajectoires de la forme, "Tragedia Endogonidia", Romeo Castellucci, Societas Raffaello Sanzio, photographies de L. Del Pia, avant-propos de R. Castelluci, trad. J.-L. Provoyeur, Arles, Actes Sud, 2008, p. I29). 
diaphragme partiellement rempli d'un liquide laiteux qui, par moments, virevolte lentement au-dessus de sa tête. De plus, soudain du sang coule de son cou, dont l'origine est cachée par la chevelure : est-elle blessée? Où? En estelle consciente? Elle semble l'ignorer et elle parait préoccupée plutôt par son apparence, puisqu'elle répète lentement le geste de se brosser les cheveux et de se regarder dans un miroir qu'elle tient à la main. Mais la menace de la mort devient de plus en plus concrète et imminente : une énorme tête de bélier soutenue par des figures habillées en blanc commence à frapper rythmiquement le rideau en visant la vieille, qui finalement succombe et se trouve couverte d'une peau de bouc par les gardes en blanc. Dans la suite du spectacle, elle va resurgir en petite fille, dont le parcours évoque le destin biologique de la femme : sur une scène dorée, une figure de démon lui fait mettre des chaussures rouges et du sang lui coule dessus ${ }^{\mathrm{I} 2}$. Le sens de la génération est ainsi bouleversé dans une boucle sans fin : finalement, donner la vie n'est rien d'autre que donner la mort ${ }^{13}$.

Dans les deux séquences d'Ève et de la malade de dos, les vieilles femmes sont immergées dans la solitude, se tenant dans des positions statiques qui semblent déterminées par des appareils et des machineries. Coupées de toute relation, les corps marqués par l'âge et la maladie, elles constituent une image en négatif du féminin et du maternel culturellement établis. En touchant les cordes les plus profondes et refoulées de l'esprit, en suscitant des réminiscences figuratives inconscientes et en déclenchant des associations oxymoriques, leur présence énigmatique, leurs gestes solennels et incompréhensibles, questionnent radicalement l'origine et la fin de la vie, et rendent palpable son caractère tragique. La vieillesse féminine est toujours porteuse d'un questionnement métaphysique mythique et absolu. Au contraire, la vieillesse masculine apparaît plutôt ancrée dans une réalité existentielle et insérée dans un réseau de relations intergénérationnelles.

I2. Sur la présence des enfants dans les créations de la Socìetas Raffaello Sanzio, voir A. Martinez, "Le Cycle de la Tragedia endogonidia, de la Societas Raffaello Sanzio : L'enfant en état de mort paradoxale», dans G. Banu (dir.), L'enfant qui meurt. Motif avec variations, Montpellier, L'Entretemps, 20I0, p. 299-306, et M. B. Lafrance, "Quand le réel entre en scène : la figure de l'enfant chez Castellucci ", dans Jeu. Revue de théâtre, n I42, 2012, p. 90-97.

13. Une autre figure de maternité niée, liée à la vieillesse, même si à notre avis de manière moins évidente par rapport à l'apparence de l'actrice, se trouve aussi dans l'épisode \#06 Paris: "Âgée est également la Mère de P.\#06 Paris, incapable de faire sortir du lait de son sein stérile mais offerte à l'union avec le dragon du carnaval chinois, union qui se révèle être une sorte d'holocauste, puisque la Mère disparait, engloutie dans le corps colossal du monstre » (E. Pitozzi et A. Sacchi, Itinera : trajectoires de la forme, "Tragedia Endogonidia», Romeo Castellucci, Societas Raffaello Sanzio, ouvr. cité, p. I30). 


\section{Pères et fils : de la métaphore à la représentation}

La tête de bélier apparaît aussi au début de Giulio Cesare (1997), inspiré de la tragédie éponyme de Shakespeare : cette fois-ci elle frappe un rideau en plastique qui se trouve face au public, directement menacé par la violence de l'histoire qu'elle évoque. Cette image déstabilisante introduit une séquence également troublante, dans laquelle le personnage de ...vski - allusion à Stanislavski insère une caméra endoscopique dans son nez et rend visible l'intérieur de son larynx, source organique de la voix, de la parole et de la rhétorique. On est ainsi plongé physiquement au cœur de l'humain, dans le mystère du langage et du pouvoir qu'il donne à celui qui le maîtrise. Des présences extrêmes - un Cicéron obèse, un Antoine ayant subi une laryngectomie, des Brutus et Cassius anorexiques ${ }^{14}$, côtoyés par un cheval et des automates - et des machineries qui modifient l'émission de la parole vont donner corps à cette interrogation sur l'homme dans un espace oppressif, enfermé par des murs de pierre, et délimité par des rideaux en plastique, qui se transforme dans la deuxième partie en un champ de ruines. La vieillesse apparait dans la première partie, lorsque Brutus et Cassius - les seuls acteurs sans caractéristiques particulières - se résolvent à tuer Jules César. Celui-ci, dans un renversement de l'image historique de l'homme politique au sommet de son pouvoir, est incarné par un vieillard's, extrêmement mince et frêle, vêtu d'une toge rouge démesurée pour son corps maigre. Cette figure immobile et silencieuse se soumet avec une docilité inconsciente, presque absente, aux manipulations de son fils adoptif. Assis sur une chaise au centre du plateau avec la tête et le buste repliés en avant, éclairé par un puits de lumière froide, il semble attendre avec résignation l'arrivée des conjurés. Lorsqu'ils s'approchent, il tourne la tête, les regarde brièvement mais immédiatement se replie et se livre à un rituel de mort paradoxal, qui mêle soin et humiliation. Brutus, qui porte un masque sanitaire, l'aide à se lever d'un geste ferme mais pas dépourvu de douceur, il le désarme, lui ôte sa toge et l'expose ainsi, debout, nu, aux regards des spectateurs, dans la même position sacrificielle d'Ėve. Avec une certaine impatience, il le rassoit et commence à le laver avec une serviette et de l'eau, avec des gestes qui suggèrent affection et habitude : la tête et le visage d'abord, ensuite les mains, les pieds. Finalement, le fils s'agenouille face au père, mais Cassius le ramène au projet homicide en lui tendant des cordes, qu'il accepte en se relevant à côté de la victime. Une suspension du geste s'ensuit, les deux conjurés imposant leurs mains sur César,

I4. Dans la deuxième partie.

I5. L'acteur est Alvaro Biserna. 
assis, immobile, la tête pliée à l'avant, comme accablé de honte. Mais ...svki les incite à poursuivre et commence à ligoter le corps fragile et souffrant du vieillard. Brutus regarde sans réagir aux plaintes de César, qui, face à ce manque de réaction, l'implore en dialecte romagnol : «Bruto, mon fils!». Pourtant, César ne se révolte pas et semble avoir accepté de jouer son rôle de victime lorsqu'il s'installe comme un modèle posant pour un tableau sur la couche préparée au sol, où il est immobilisé par un réseau de lacets. Pendant cette opération, retentissent dans l'espace des voix joyeuses d'enfants en train de jouer. Et c'est aussi avec une voix surprenante et incohérente d'enfant - obtenue grâce à l'inhalation du gaz hélium - que Brutus entame le discours visant à justifier son action, dictée par l'opportunité politique et exécutée malgré son amour pour César. L'action scénique suggère ainsi un lien entre parricide et passage à l'âge adulte. Le devenir adulte, imposé par l'Histoire, caractérisé par le dépassement incomplet d'une condition enfantine et par la volonté d'anéantir le père, se situe dans un entre-deux irrésolu, incommode et velléitaire. Le vieillard, avec sa passivité et sa fragilité, semble au contraire se placer au-delà de l'Histoire, dans un état intemporel qui anticipe l'atonie et la perte de soi de la mort.

Des éléments gestuels et sonores de cette séquence reviennent dans un spectacle postérieur, Sul concetto di volto nel Figlio di Dio (2OII), dans lequel la relation entre enfance, âge adulte et vieillesse est centrale. Ils sont néanmoins insérés dans un contexte scénique radicalement différent, à la fois plus humain et plus sacré. Comme dans Giulio Cesare, les figures de ce spectacle sont toutes masculines : un homme adulte, actif, comme l'indiquent son costume et sa mallette; son père, âgé et malade; un groupe d'enfants se réunissant progressivement, peut-être à la sortie de l'école ou de la salle de sport; et, dominant l'espace du fond de scène et en position symétrique par rapport au public, le jeune visage du Christ Salvator Mundi peint par Antonello da Messina (I465-I475 env.). La création - qui avait suscité en France de vives protestations de la part de groupes intégristes catholiques en raison du caractère supposément blasphématoire des atteintes portées à l'image de Jésus - est organisée en trois parties. Dans la première, d'environ trente minutes, le père et le fils sont les protagonistes d'une véritable passion, tandis que Jésus constitue une sorte de spectateur muet, suspendu entre présence et absence; dans la deuxième, d'environ dix minutes, séparée de la première par un bref intermède dans lequel le noir est animé par un tissu sonore de vagues, souffles, chutes rythmiques et répétées d'objets, les enfants défient Jésus par une sorte de révolte impossible de l'innocence, en jetant des grenades contre le portrait, tandis que le père reste immobile, assis côté cour, absent et présent à la fois, tout comme le Christ dans la partie précédente. L'attaque terminée, les enfants partent d'abord; ensuite le vieillard resté seul sort aussi de scène, en traversant en diagonale le plateau couvert de grenades. Commence alors 
la séquence finale, d'une dizaine de minutes, dans laquelle le portrait devient l'objet de transformations, de violences, d'interrogations plastiques et écrites, dans un environnement sonore dense et aigu de lamentations, de bruits stridents et menaçants, auxquels succèdent, vers la fin, des chants d'oiseaux apaisés.

La vieillesse constitue ici le germe d'un questionnement profond sur la compassion, la patience, la souffrance. Elle ne constitue plus, comme dans Giulio Cesare, un faisceau complexe de sens multiples, où des fonctions ostentatoires, métaphoriques et synecdotiques se mêlent pour évoquer une interprétation perturbante du geste homicide de Brutus et donner une vision cruelle des rapports de pouvoir dans l'histoire. Ici, elle est l'objet d'une observation directe, sans médiations, et finalement d'une véritable représentation. Dans la première partie du spectacle, Castellucci place violemment face aux spectateurs le double calvaire d'un homme trahi par son corps et du fils qui l'assiste malgré tout. Dans un décor hyperréaliste composé de meubles blancs au design minimaliste se produisent une chute dans l'abjection physique et une mise à l'épreuve extrême de l'amour, scéniquement organisées comme une traversée du plateau. Tout commence lorsque le fils s'apprête à dire au revoir au père malade, assis sur le canapé en train de regarder la télé, après lui avoir laissé des médicaments et lui avoir caressé la tête. Juste avant qu'il ne s'en aille, il s'aperçoit que le père vient de déféquer - il lui demande "L'as-tu fait?» comme on le demanderait à un enfant - et qu'il faut le changer. Il accomplit alors les mêmes gestes que Brutus : il le fait lever, lui ôte ses habits de malade et va chercher les outils pour le nettoyer - des seaux, des serviettes, des gants en plastique, des couches propres. Lorsqu'il a terminé et s'apprête à partir, l'accident se répète, cinq fois de suite, de manière de plus en plus accélérée, dans un mouvement scénique vers la droite - du salon au lit, en passant par la salle à manger - qui synthétise en une demi-heure l'évolution terrible de la maladie. À chaque «station», le vieillard, comme déjà Ėve et César, reste debout dans sa solitude, face ou plus souvent dos au public, nu, incapable de cacher sa dégradation non seulement aux regards des spectateurs, mais aussi à celui du Christ. Face à son fils, il est docile et passif comme César, mais montre aussi le malaise profond provoqué par la conscience de son état. L'acteur ne se limite pas à porter la honte d'être sur scène par sa seule présence, mais joue véritablement la condition du personnage, par des gestes de désespoir - se cacher le visage derrière les mains, par exemple -, des lamentations, des pleurs. Le fils persiste dans ses soins affectueux en essayant de dissimuler le dégoût, l'impatience, l'impuissance, la fatigue, face à ce corps de père réduit à un état organique incontrôlable, mettant à l'épreuve l'amour filial le plus solide. Le public est lui aussi mis à l'épreuve et conduit dans les territoires de l'intolérable : à travers la vue et l'ouïe, parce qu'il assiste à des situations intimes, vraies, connues, dont on voudrait oublier l'existence et l'expérience; et à travers 
l'odorat, puisque une puanteur d'excréments se répand progressivement et enveloppe les spectateurs, en anéantissant toute distance entre la scène et la salle. Avec son regard immobile, imperturbable et serein, qui ne donne que par moments l'illusion d'être vivant grâce à des variations lumineuses, le Christ accentue la circulation des rôles, des émotions, des questionnements : placé face au public, comme un miroir grandissant de chaque spectateur, le portrait constitue d'une part un double de la fonction spectatorielle, qui met en question les réactions du public; de l'autre, sa distance - ontologique et émotionnelle - resserre les liens entre le fils, le vieillard et les spectateurs, qui se retrouvent ensemble à scruter le mystère de cette divinité, à l'implorer, à l'interroger sur la condition humaine. Lorsqu’à la fin de la séquence le fils, désemparé, s’approche du portrait et essaie de l'embrasser, son geste semble soutenu et porté par la salle tout entière.

Les enfants, qui représentent les débuts insouciants et innocents de la vie, destinée à se terminer sur ce lit de maladie et de mort où le vieillard reste assis, sont aussi interpellés par le visage de Christ et l'agressent physiquement. Leur révolte ne parvient pas non plus à faire réagir cette divinité à la fois si humaine et si distante; pas plus que les actions du créateur-metteur sur l'image et sur la toile qui l'accueille en scène ne parviennent à la modifier. Le spectacle s'achève sur la phrase "You are (not) my sheperd» (Tu [n']es [pas] mon pasteur), où la négation paraît et disparaît, en laissant le spectateur sans réponse, seul avec ses doutes et ses questionnements.

Dans ce spectacle, la vieillesse devient un moment spécifique de la vie à explorer dans ses conséquences concrètes et dans son rapport avec les autres générations. Elle demeure aussi le catalyseur d'une réflexion existentielle et métaphysique, plongée néanmoins dans le présent de notre monde. Dans le jeu de miroirs entre scène et salle, le dispositif théâtral aussi est mis en question. Le portrait du Christ, qui demeure serein et impassible face à la dégradation et à la souffrance, dédouble et interroge le regard du public et évoque la figure du metteur en scène (créateur invisible de ce lieu de l'insupportable, centré sur la réalité du corps exhibé sur scène, sur les signes de sa vieillesse). Comment se situer entre réel et représentation? Pourquoi regarder? Et comment?

\section{Figurants de l'homme, figurants de l'acteur}

La tension irrésolue et perturbante entre réalité et théâtre s'incarne aussi dans les déclinaisons variables du "figurant", le non-acteur que Castellucci extrait du monde et expose sur la scène : celui-ci sert d'abord à rendre présent l'homme, mais aussi à rendre visible la fonction de l'acteur en tant que porteur d'une transfiguration théâtrale déployée par des «masques» - costume, maquillage, 
accessoires, coiffures - qui le déterminent dans son altérité radicale. Après les corps violemment réels dans leur caractère exceptionnel des créations des années 1990 et 2000 , des figures d'hommes ordinaires ont fait leur apparition sur scène, parfois en tant que personnages joués par des acteurs-interprètes, comme dans le cas du fils et du vieillard de Il concetto di volto, parfois en tant que foules anonymes. Dans Inferno (2008), cinquante figurants - dont seulement un noyau de professionnels - évoquent la multitude des damnés dans l'au-delà et l'humanité tout entière, aux prises avec les contradictions, les violences et les souffrances de la vie. Dans la composition de ce double du public, formé d'individus habillés hauts en couleurs, accomplissant des gestes répétés - tomber au sol, se lancer dans le vide, donner des coups de pied sur le mur du fond - l'âge constitue un critère fondamental. Toutes les générations sont représentées : des bébés, des enfants, des adolescents, des adultes, des vieillards, tous définis non pas par leurs caractères individuels, mais par leurs liens familiaux et par leur place dans le réseau de relations parentales au fondement de la société humaine. Fils, partenaires, parents, grands-parents s'aiment, se repoussent, se débattent, s'entretuent. Deux personnes âgées, un homme et une femme, jouées par Maria Luisa Cantarelli et Gianni Plazzi ${ }^{16}$, se détachent clairement de l'ensemble. Après que les damnés se sont rassemblés sur scène, le vieillard sort du rang et se place face aux spectateurs, debout, seul, tenant entre les mains le ballon de basket avec lequel un enfant vient de jouer. Après quelques instants, un autre damné vient le rejoindre et lui prend le ballon. Le vieillard se retire. Le relais est ensuite pris à tour de rôle par les autres damnés, et le ballon passe calmement de mains en mains six fois jusqu'à arriver entre celles de la femme, qui, après un moment de suspension, commence à le mordre avec une voracité animale et à émettre des bruits de cochon, en évoquant ainsi l'image horrifiante qui conclut la rencontre de Dante avec le conte Ugolino, dans le cercle des traîtres ${ }^{17}$. Le couple de vieillards, qui ouvre et clôt le cycle d'appropriation et d'expropriation, concentre dans ses comportements l'ambiguïté de l'être humain, victime d'une avidité qui apparait comme acceptée et inévitable - le silence et le calme de leurs attitudes le suggèrent - mais qui conduit à la

I6. Il s'agit des interprètes d'Ève dans Genesi et de la vieille dans \#0s Bergen, et du vieillard dans Sul concerto di volto bel figlio di Dio, donc de deux figures importantes pour la compagnie, dont la présence s'insère dans le cadre autoréflexif du spectacle, posé au début par l'apparition sur le plateau du metteur en scène se présentant au public ("Je m’appelle Romeo Castellucci») se donnant en sacrifice à des chiens enragés (tout en portant bien sûr les protections adaptées), et déployé par la suite par la dédicace aux acteurs décédés de la Raffaello Sanzio, projetée sur le mur de fond.

I7. "Quand il eut dit ces mots, le regard tors,

il reprit le malheureux crâne avec ses dents, qui mordirent l'os, comme celle d'un chien.»

(Dante, La Divine Comédie, L'Enfer, trad. J. Risset, Paris, Flammarion, I992, XXXIII, v. 75-78, p. 299.) 
perte de soi et de son humanité. Le même schéma se reproduit tout au long du spectacle, et l'homme et la femme interviennent au début et à la fin des séquences d'actions - évoquant vaguement les péchés de Dante - accomplies pas l'ensemble des figurants. La dernière, avant qu'ils soient tous enfermés dans les voûtes dans le mur de fond comme les cadavres dans une morgue, est celle de l'assassinat : un à un, les damnés s'approchent d'un partenaire, l'embrassent et ensuite font le geste de l'égorger, et leur victime tombe alors par terre. Finalement, il ne reste sur scène que les deux vieillards. L'homme tue la femme, il regarde les cadavres allongés sur le plateau, et s'exclame «Où es-tu? Je t'implore.» Entre alors un enfant. Les deux s'embrassent tendrement, et ensuite, calmement et sans hésitation, l'enfant tue le vieillard. La boucle est bouclée, et l'enfant, malgré son innocence et son inconscience, est prêt à recommencer le cycle d'amour et de haine, de fragilité, de cruauté et de désespoir, dans laquelle l'humanité est enfermée, dont les vieillards portent le poids, les enfants le destin. L'utilisation des deux extrêmes de la vie assume dans Inferno une valeur inclusive, elle sert à montrer l'impossibilité d'échapper à l'enfer intrinsèque à la condition humaine. Ces «figurants de l'homme» produisent un effet d'identification avec les spectateurs, une identification toujours flottante et individuelle, fondée sur les expériences et les ressentis de chacun, mais aussi sur un socle de vérité commune ancré dans l'existence.

Leur caractère ordinaire s'oppose aux "figurants de l'acteur", qui apparaissent dans d'autres créations en tant que présences solitaires et isolées mettant en scène la tradition théâtrale et ses masques, comme, dans la Tragedia Endogonidia, les "auxiliaires» et "commutateurs d'événements ${ }^{18}{ }^{8}$ représentés par l'Arlequin de \#07 Roma et le Clown de \#02 Avignon. Ce dernier est une figure grotesque de clown blanc, avec son costume blanc à paillettes, son chapeau pointu et son maquillage blanc et rouge, une figure agile et légère, mais au visage marqué par la vieillesse. Comme un cambrioleur, il descend sur la scène par une fenêtre et commence à passer sur le sol, également d'une blancheur immaculée, une serpillière, qui est par contre constituée d'un gros foie d'animal trempé dans un seau d'eau sanglante. Il apparaît par la suite presque nu, avec seulement ses bas et ses chaussures blanches, avec un phallus en caoutchouc sur la poitrine, pour se glisser dans une housse blanche et se détendre sur un matelas. Son aspect, ses comportements et ses actions multiplient les contradictions énigmatiques, illisibles, qui peuvent être pensées comme les symptômes d'une transformation théâtrale inachevée, incapable de dépasser les limites de la réalité. L'union du masque du clown avec le visage visible du vieillard montre ce que l'acteur ne peut pas faire : transcender

18. E. Pitozzi et A. Sacchi, Itinera: trajectoires de la forme, "Tragedia Endogonidia", Romeo Castellucci, Societas Raffaello Sanzio, ouvr. cité, p. I63-166. 
sa dimension temporelle. Son sacrifice scénique s'enrichit d'un sens tragique : dans ce cas, la vulnérabilité de l'homme et sa mortalité semblent contaminer le masque et contredire le pouvoir de métamorphose du théâtre.

Si dans le théâtre de la Socìetas Raffaello Sanzio et de Romeo Castellucci la figure de l'acteur vieillissant garde donc un élément d'artificialité, de mise en scène, elle assume pleinement son sens tragique lorsqu'on se trouve face à l'acteur et non pas à son «figurant». Dans le théâtre italien, encore fortement lié à la tradition du grand acteur, nombreuses sont les incarnations de cette vieillesse d'acteur authentique, souvent involontaire, sinon refoulée : les joues creusées d'Eduardo De Filippo, le corps agile mais exténué de Ferruccio Soleri, l'Arlequin de Giorgio Strehler, les tremblements de Dario Fo... C'est Carmelo Bene qui a déployé le sens tragique de la vieillesse de l'acteur de la manière la plus intéressante, en l'insérant dans son travail sur le visage et le masque. Dans Pinocchio, sa dernière création télévisuelle inspirée du spectacle éponyme de I998, l'acteur joue le pantin protagoniste et donne sa voix - enregistrée et modifiée par des technologies phoniques complexes - à tous les autres personnages, joués visuellement par une même actrice, Sonia Bergamasco grâce à plusieurs masques ${ }^{19}$. Dans le déploiement audiovisuel de cet univers de déguisements, déformations et écarts entre identités sonores et visuelles, où les acteurs subissent un processus radical de déshumanisation, la caméra se concentre souvent sur le gros plan de Bene-Pinocchio : sa peau est couverte d'un épais maquillage qui crée un effet de bois, les yeux sont cernés au crayon noir, un faux nez déforme ses traits, sa pose est oblique et manque de naturel. Néanmoins, ces artifices laissent transparaître le visage de l'acteur, marqué par l'âge et par la maladie : derrière le pantin à l'uniforme d'écolier apparaît alors le vieillard malade. Il n'est plus question, comme sur le vase de Pronomos de mettre en scène la dimension temporelle de l'homme par des masques et des costumes spécifiques. C'est plutôt, chez Carmelo Bene, comme dans les créations de la Socìetas Raffaello Sanzio, le temps qui intervient directement dans le périmètre du théâtre en produisant des brèches dans les masques, en laissant percevoir les hommes qui les portent. La vieillesse, plus que la mort, devient une marque révélatrice de l'humanité du théâtre, de sa vérité et de ses limites.

19. La dramaturgie sonore de Pinocchio et sa relation à l'espace ont été récemment analysées par C. De Simone dans sa communication «Pinocchio de Carmelo Bene : un spectacle de marionnettes à la radio" au colloque L'Idée d'espace "sensible» dans la dramaturgie musicale, université Paris 8 - Labex Arts $\mathrm{H}_{2} \mathrm{H}$, IO-II avril 20I4. Voir aussi la partie finale de l'article de C. De Simone, «Ventiloquies», dans C. De Simone et C. Biet (dir.), d'après C. Bene, Revue d'Histoire du Théâtre, n 263, juillet-septembre 2014, p. 295-306. 